\title{
Numerical Simulation of Reciprocating Flow Forced Convection in Two-Dimensional Channels
}

\author{
Cuneyt Sert \\ Ali Beskok \\ e-mail: abeskok@mengr.tamu.edu \\ Mechanical Engineering Department, \\ Texas A\&M University, \\ College Station, TX 77840-3123
}

\begin{abstract}
Numerical simulations of laminar, forced convection heat transfer for reciprocating, twodimensional channel flows are performed as a function of the penetration length. Womersley $(\alpha)$ and Prandtl (Pr) numbers. The numerical algorithm is based on a spectral element formulation, which enables high-order spatial resolution with exponential decay of discretization errors, and second-order time-accuracy. Uniform heat flux and constant temperature boundary conditions are imposed on certain regions of the top surface, while the bottom surface is kept insulated. Periodicity of velocity and temperature fields is imposed on the side boundaries, while the flow is driven by an oscillating pressure gradient. These sets of boundary conditions enable time-periodic solution of the problem. Instantaneous and time-averaged surface and bulk temperature distributions, and Nusselt number variations are presented. For high $\alpha$ flows, the temperature field is significantly affected by the Richardson's annular effect. Overall, forced convection increases by increasing the penetration length, $\alpha$ and Pr. Corresponding steady-flow simulations are performed by matching the volumetric flowrate. For the limited parameter space investigated in this paper, steady unidirectional forced convection is more effective than the reciprocating flow forced convection. [DOI: 10.1115/1.1565092]
\end{abstract}

Keywords: Channel Flow, Computational, Forced Convection, Heat Transfer, Pulsating

\section{Introduction}

Fluid flow and heat transfer in circular tubes, ducts and channels have extensive engineering applications, including heat exchanger design, biomedical engineering and micro-fluidics. Steady forced convection heat transfer in channels and tubes is well understood. Simple geometry and steady flow conditions enable analytical solutions and collection of reliable experimental data. This results in analytical and empirical relations for the Nusselt number variations in terms of the flow parameters (Shah and London [1]). However, there are relatively fewer investigations of oscillatory-flow heat transfer, which has more stringent time and spatial resolution requirements. Oscillatory flows can be grouped into two categories: pulsating (modulated) and reciprocating (fully reversing) flows. Pulsating flows are always unidirectional and can be decomposed into steady and unsteady components, such as in the case of blood flow in arteries (Zamir [2]). For reciprocating flows, the flow direction changes cyclically. Hence, these flows convect zero net mass. With the advent of microelectromechanical systems (MEMS) and micro-fluidics, pulsating and reciprocating flows are finding more engineering applications. For example, membrane driven micro-pumps and peristaltic micro-mixers result in pulsating flows (Beskok and Warburton [3], Yi et al. [4]). Several novel heat exchanger devices for electronic cooling applications utilize reciprocating flow and heat transfer (Liao et al. [5], Sert and Beskok [6]). Reciprocating flows are also utilized to enhance mixing in micro-scales (Oddy et al. [7], Dutta and Beskok [8]).

Analyses of pulsating and reciprocating flow heat transfer differ from each other, mainly due to the thermal and velocity boundary conditions. Pulsating flows are unidirectional. Hence, they have

Contributed by the Heat Transfer Division for publication in the JOURNAL OF HEAT TRANSFER. Manuscript received by the Heat Transfer Division January 14, 2002; revision received December 4, 2002. Associate Editor: S. P. Vanka. permanent inflow and outflow regions, where one can easily define the inlet velocity and temperature boundary conditions. Reciprocating flows require interchange between the inflow and outflow boundaries during a cycle. For most applications, it is difficult to determine the inflow/outflow boundary conditions, since fluid particles exiting the flow domain during a part of the cycle are fed back into the domain, later in the cycle. Although the momentum equation yields an analytical solution for twodimensional fully developed reciprocating channel flows, analytical solution of the heat transfer problem is not possible, unless the thermal boundary conditions are simplified. In this paper, we mainly concentrate on reciprocating flow heat transfer. Hence, we will discuss the previous work on reciprocating flow heat transfer in detail. Some experimental, numerical and analytical studies on pulsating flow heat transfer can be found in (Siegel and Perlmutter [9]; Siegel [10]; Kim et al. [11]; Moschandreou and Zamir [12]; Zhao and Cheng [13]; Greiner et al. [14]).

A literature survey on reciprocating flow heat transfer shows two different categories of investigations. The first one is focused on heat conduction enhancement with high frequency, low amplitude oscillations, while the second one is focused on forced convection with low frequency, large amplitude oscillations in relatively short channels. One of the early studies of the former category is due to Chatwin [15], who showed enhancement of species diffusion under high frequency oscillations. Later this phenomenon was applied to enhance heat transfer, where effective thermal diffusivities, that are about three orders of magnitudes higher than the values due to molecular thermal diffusion, are achieved (Kurzweg and Zhao [16]; Kurzweg [17]).

Regarding the second category, Li and Yang [18] investigated heat transfer in reciprocating flows at low frequencies and large amplitudes by numerical simulations. They showed heat transfer enhancement due to the intra-cycle oscillations, which were caused by "sudden changes of the inlet and exit boundary conditions." Liao et al. [5] performed forced-convection experiments 


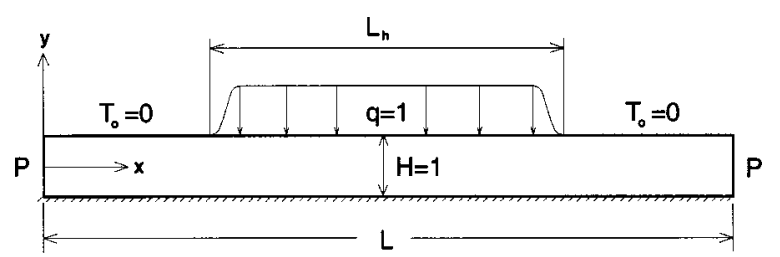

Fig. 1 The geometry and thermal boundary conditions used in this study. On the top surface, uniform heat flux of $q=1$ is specified at $5 \leqslant x \leqslant 15$. For $4 \leqslant x \leqslant 5$ and $16 \geqslant x \geqslant 15$, the heat flux varies from zero to unity sinusoidally. Zero wall temperature is specified for $x \leqslant 4$ and $x \geqslant 16$. Bottom wall is insulated, while side surfaces are periodic (cyclic/repeating).

on microprocessor chips by means of channeled zero-mean oscillatory air flow. They measured the surface temperature of several power generating components in a typical personal computer, and reported heat transfer enhancement by oscillatory forced convection, compared to the conventional fan cooling. Based on the Reynolds number (Re), Liao et al. [5] distinguished two different heat transfer enhancement mechanisms. In the low Re regime, heat transfer enhancement was observed due to the reduction in the Stokes layer thickness with increased flow frequency. While for the high Re regime, heat transfer enhancement was observed due to the presence of "higher-order harmonics of imposed flow frequency." Cooper et al. [19] investigated forced convection heat transfer by heating the bottom wall section of a rectangular duct. Experiments were performed at low frequencies with large tidal displacements. The results showed enhanced heat transfer rates for increased oscillation frequencies and tidal displacements, and decreased duct heights. Chou et al. [20] used oscillatory flow to cool electronic devices. Their idea was to carry the heat away from the source using bubbles oscillating in a micro channel. Preliminary results show heat transfer enhancement caused by the oscillatory flow.

In this study, we present numerical solutions of reciprocating fluid flow and heat transfer in two-dimensional channels. This paper is organized as follows. First, we further define the problem, and state the boundary conditions. Then the governing equations are presented, followed by the exact solution of the fluid flow problem and demonstration of numerical convergence characteristics of our scheme for high Womersley number flows. Finally, the temperature field results are presented and the effects of various flow and heat transfer parameters on time-periodic oscillatory forced convection are discussed.

\section{Problem Definition and Important Parameters}

A schematic view of the computational domain along with the associated boundary conditions is shown in Fig. 1. We consider fully reversing flow driven by an oscillatory pressure gradient. The middle portion of the top plate is uniformly heated, while its two sides are kept at constant temperature, and the bottom plate is insulated. The presence of constant temperature zones allows a time-periodic solution for the heat transfer problem. Periodic (cyclic/repeating) boundary conditions are specified at the two ends. The periodicity condition is such that fluid coming out of one side enters through the other side. Therefore, the velocity and temperature values are always the same at both ends. For a better understanding of the periodic boundary conditions, this problem can be visualized as a portion of an infinitely long channel with repeated constant temperature and constant heat flux sections as shown in Fig. 2. Such a configuration can be observed in electronic cooling applications, where the IC boards usually have a repeating pattern. For this application, our analysis would be valid sufficiently away from the device inlet/exit regions, where flow development effects are negligible.

In our simplified two-dimensional model, there are four important geometric length scales: channel height $H^{*}$, total channel

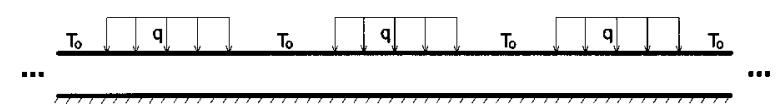

Fig. 2 Schematic view of a hypothetical problem that consists of a channel with repeating heated and constant temperature boundaries

length $L^{*}$, length of the heated portion of the channel $L_{h}^{*}$, where heat flux is applied, and the penetration length $L_{p}^{*}$ (tidal displacement). Penetration length is the average distance traveled by fluid particles during one-half of an oscillation period $\left(\tau^{*} / 2\right)$. Considering sinusoidal oscillations, the penetration length is defined as

$$
L_{p}^{*}=\bar{u}^{*} \frac{\pi}{\omega^{*}}
$$

where $\omega^{*}=2 \pi / \tau^{*}$ is the oscillation frequency and $\overline{\bar{u}}^{*}$ is the time and cross-channel averaged axial velocity. The parameter $L_{p}^{*}$ is a practical measure of the oscillation amplitude. For efficient cooling, $L_{p}^{*}$ should be large enough so that the heated fluid under the constant heat-flux region will travel towards the constant temperature boundaries, where efficient heat transfer to the surroundings can take place.

For oscillatory flows the Womersley number is an important non-dimensional parameter, defined as

$$
\alpha=\sqrt{\frac{\omega^{*} H^{* 2}}{\nu^{*}}} .
$$

The Womersley number determines the velocity profile. Small $\alpha$ values result in a quasi-steady flow with oscillatory parabolic velocity profiles. However, large $\alpha$ values lead to the well-known "Richardson's annular effect" that results in near-wall velocity overshoots, where the maximum velocity no longer occurs at the symmetry plane (Zhao and Cheng [13]; Richardson and Tyler [21]). This has direct effects on heat transfer, since high velocities with large gradients increase the heat removal rate from the surfaces. Zhao and Cheng reported observing annular effects in the temperature profiles [13]. This is also verified in our current study. The Womersley number is sometimes called the "kinetic Reynolds number" because it plays the same role as the Reynolds number in unidirectional steady flows. The Prandtl number (ratio of momentum and thermal diffusivities) is also important in heat transfer. For reciprocating flows, the thermal boundary layer thickness is determined by both the Prandtl and Womersley numbers.

Selecting the channel height $H^{*}$ as the characteristic length scale, the important nondimensional parameters are $L$ $\left(=L^{*} / H^{*}\right), L_{h}\left(=L_{h}^{*} / H^{*}\right), L_{p}\left(=L_{p}^{*} / H^{*}\right), \alpha$ and Pr. This fiveparameter space makes it difficult to study the importance of every parameter in detail. Therefore, we fixed the normalized channel length $L$ and the heated region length $L_{h}$, and varied $L_{p}$, $\alpha$, and Pr, by using two different values for each of these parameters. This results in eight different conditions, which are summa-

Table 1 Non-dimensional parameters used in the simulations

\begin{tabular}{ccccccc}
\hline \hline Case no. & $L$ & $L_{h}$ & $L_{p}$ & $\alpha$ & $\operatorname{Pr}$ & $\operatorname{Re}^{\prime}$ \\
\hline 1 & 20 & 12 & 5 & 1 & 1 & $5 / \pi$ \\
2 & 20 & 12 & 5 & 1 & 10 & $5 / \pi$ \\
3 & 20 & 12 & 5 & 10 & 1 & $500 / \pi$ \\
4 & 20 & 12 & 5 & 10 & 10 & $500 / \pi$ \\
5 & 20 & 12 & 10 & 1 & 1 & $10 / \pi$ \\
6 & 20 & 12 & 10 & 1 & 10 & $10 / \pi$ \\
7 & 20 & 12 & 10 & 10 & 1 & $1000 / \pi$ \\
8 & 20 & 12 & 10 & 10 & 10 & $1000 / \pi$ \\
\hline \hline
\end{tabular}


rized in Table 1 . The parameters appearing in Table 1 are all nondimensional.

In our simulations the input parameters are $L_{p}, \alpha$, and Pr. The flow is driven by an oscillatory pressure gradient given by,

$$
\frac{\partial p^{*}}{\partial x^{*}}=-A^{*} \cos \left(\omega^{*} t^{*}\right)
$$

where $p^{*}, A^{*}$, and $t^{*}$ are the pressure, pressure gradient amplitude and time, respectively. The amplitude $A^{*}$ is directly related to $\overline{\bar{u}}^{*}$ introduced in Eq. (1). Its value is calculated using the analytical velocity profile, which will be demonstrated in the forthcoming sections.

\section{Nondimensionalization and Governing Equations}

Numerical simulations are performed using nondimensional parameters. The length, time, velocity, pressure, temperature and heat flux are normalized as follows:

$$
\begin{gathered}
x=\frac{x^{*}}{H^{*}} \quad y=\frac{y^{*}}{H^{*}} \quad t=\frac{t^{*}}{1 / \omega^{*}} \quad u=\frac{u^{*}}{\omega^{*} H^{*}} \\
p=\frac{p^{*}}{\rho^{*}\left(\omega^{*} H^{*}\right)^{2}} \quad T=\frac{T^{*}-T_{o}^{*}}{\Delta T^{*}} \quad q=\frac{q^{*}}{k^{*} \Delta T^{*} / H^{*}}=1,
\end{gathered}
$$

where the velocity is normalized by $\omega^{*} H^{*}$ due to the lack of a characteristic velocity scale in the problem. In Eq. (4), $T^{*}$ and $q^{*}$ represent the temperature and heat flux, respectively, while the specified wall temperature is $T_{o}^{*}$, and $\Delta T^{*}$ is a reference temperature difference in the domain. Since there is only one reference temperature value on the wall $\left(T_{o}^{*}\right)$ and uniform or zero heat flux conditions are specified on the rest of the boundary, $\Delta T^{*}$ is determined in the post-processing stage. We calculate the appropriate value for $\Delta T^{*}$ using the maximum allowable temperature difference in the flow domain (based on the design considerations) and the calculated maximum nondimensional temperature $\left(T_{\max }\right)$. As an example, lets consider an electronic cooling application, where the maximum temperature difference between the ambient and the chip surface is $30^{\circ} \mathrm{C}$. If our simulation results in $T_{\max }$ $=2$, then $\Delta T^{*}=15^{\circ} \mathrm{C}$. We can calculate the maximum possible heat dissipation $\left(q^{*}\right)$ from the system using $q^{*}=q k \Delta T^{*} / H^{*}$. Alternatively, one can select the desired heat flux $\left(q^{*}\right)$, and calculate $\Delta T^{*}$ to find the maximum surface temperature. This normalization makes it easier to utilize dynamic similarity for obtaining the dimensional temperature and heat flux values.

Governing equations are the conservation of mass, incompressible Navier-Stokes and heat transport equations, presented in the following nondimensional form,

$$
\begin{gathered}
\nabla \cdot \vec{u}=0, \\
\frac{\partial \vec{u}}{\partial t}+(\vec{u} \cdot \nabla) \vec{u}=-\nabla p+\frac{1}{\operatorname{Re}} \nabla^{2} \vec{u}, \\
\frac{\partial T}{\partial t}+(\vec{u} \cdot \nabla) T=\frac{1}{\mathrm{Pe}} \nabla^{2} T,
\end{gathered}
$$

where $\operatorname{Re}=\alpha^{2}$ for oscillatory flows. Thermal conduction coefficient and viscosity are assumed to be constant and the viscous heating terms in the heat transport equation are neglected. These assumptions and approximations are consistent with the previous analytical and numerical studies.

At this point it is worthwhile to mention that the flow inside the channel is hydrodynamically fully developed (i.e., the streamwise gradient of the velocity vector is zero) at all times. Based on Fig. 2 and the discussion about the periodic end conditions, fullydeveloped flow is automatically satisfied. Therefore, the entry and flow development effects are excluded in the current study.

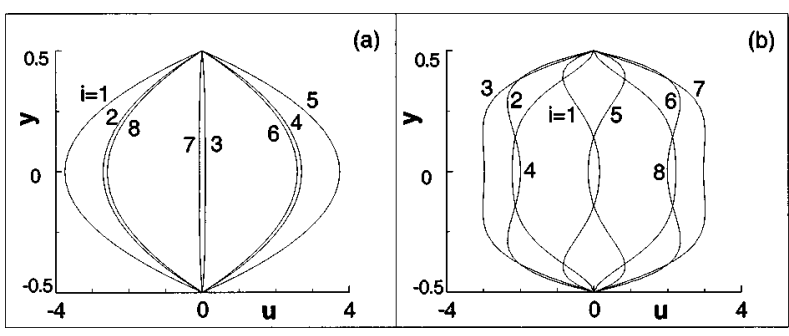

Fig. 3 Analytical solution of the velocity profiles at various times during a cycle for (a) $\alpha=1$, and (b) $\alpha=10$ flow. Index $i$ represents time within a period of the pressure pulse $(t=i$ $-1 / 8 \tau)$.

\section{Analytical Solution of the Velocity Field}

The analytical solution of reciprocating flows in a twodimensional channel is known. Consider the flow between two parallel plates, driven harmonically in time with a pressure gradient of the following form,

$$
-\frac{\partial p}{\partial x}=A e^{-i t},
$$

where $A$ is the pressure gradient normalized with $\left(\rho^{*} \omega^{* 2} H^{* 3}\right)$, and $i=\sqrt{-1}$. The velocity profile for this flow is given by (Landau and Lifshitz [22])

$$
u(y, t)=\operatorname{Real}\left\{i A e^{-i t}\left[1-\frac{\cos \left(\frac{(i+1) \sqrt{2}}{\alpha} y\right)}{\cos \left(\frac{(i+1)}{\sqrt{2} \alpha}\right)}\right]\right\}
$$

where $y$ is the cross channel distance normalized by the channel height $H^{*}$. Figure 3 shows the velocity profiles at various instances during a cycle for $\alpha=1$ and $\alpha=10$ flows. Quasi-steady flow behavior is observed for $\alpha=1$ flow, while the Richardson's annular effect is present for $\alpha=10$.

The time and cross-channel-averaged velocity $(\overline{\bar{u}})$ is obtained by integrating Eq. (9) as follows,

$$
\overline{\bar{u}}=\frac{1}{\tau H} \int_{0}^{\tau} \int_{-H / 2}^{H / 2} u(y, t) d y d t,
$$

where $\tau=2 \pi$ (radians) is the normalized oscillation period, and $H=1$ is the normalized channel height. This integral is evaluated numerically, and $\overline{\bar{u}}=0.0528 \mathrm{~A}$ and $\overline{\bar{u}}=0.5647 \mathrm{~A}$ are obtained for $\alpha=1$ and $\alpha=10$, respectively. For a desired tidal displacement $L_{p}$, we calculate the corresponding pressure amplitude $A$, using $\overline{\bar{u}}=L_{p} / \pi$, which is a nondimensional form of Eq. (1).

\section{Numerical Accuracy and Convergence}

Our numerical algorithm utilizes spectral element discretization of two-dimensional, unsteady Navier-Stokes and heat transport equations (Eqs. (5-7)). Time integration is handled by a secondorder accurate, stiffly stable time integration scheme. In this section, we demonstrate the space and time accuracy of our numerical solution by comparisons with the analytical solution of the reciprocating flow velocity profile. Simulations were performed using 104 elements, where 26 elements were employed in the streamwise direction and 4 elements were employed in the cross flow direction, as shown in Fig. 4 (top). In the spectral element method, we keep the elemental discretization of the domain fixed, and refine our solution by increasing the expansion order within each element ( $p$-type refinement). In Fig. 4 (bottom), we show quadrature points obtained by $p$-type refinements, which result in $16,49,100$, and 169 collocation points for $3^{\text {rd }}, 6^{\text {th }}, 9^{\text {th }}$, and $12^{\text {th }}$ order spectral expansions, respectively. The spectral element 

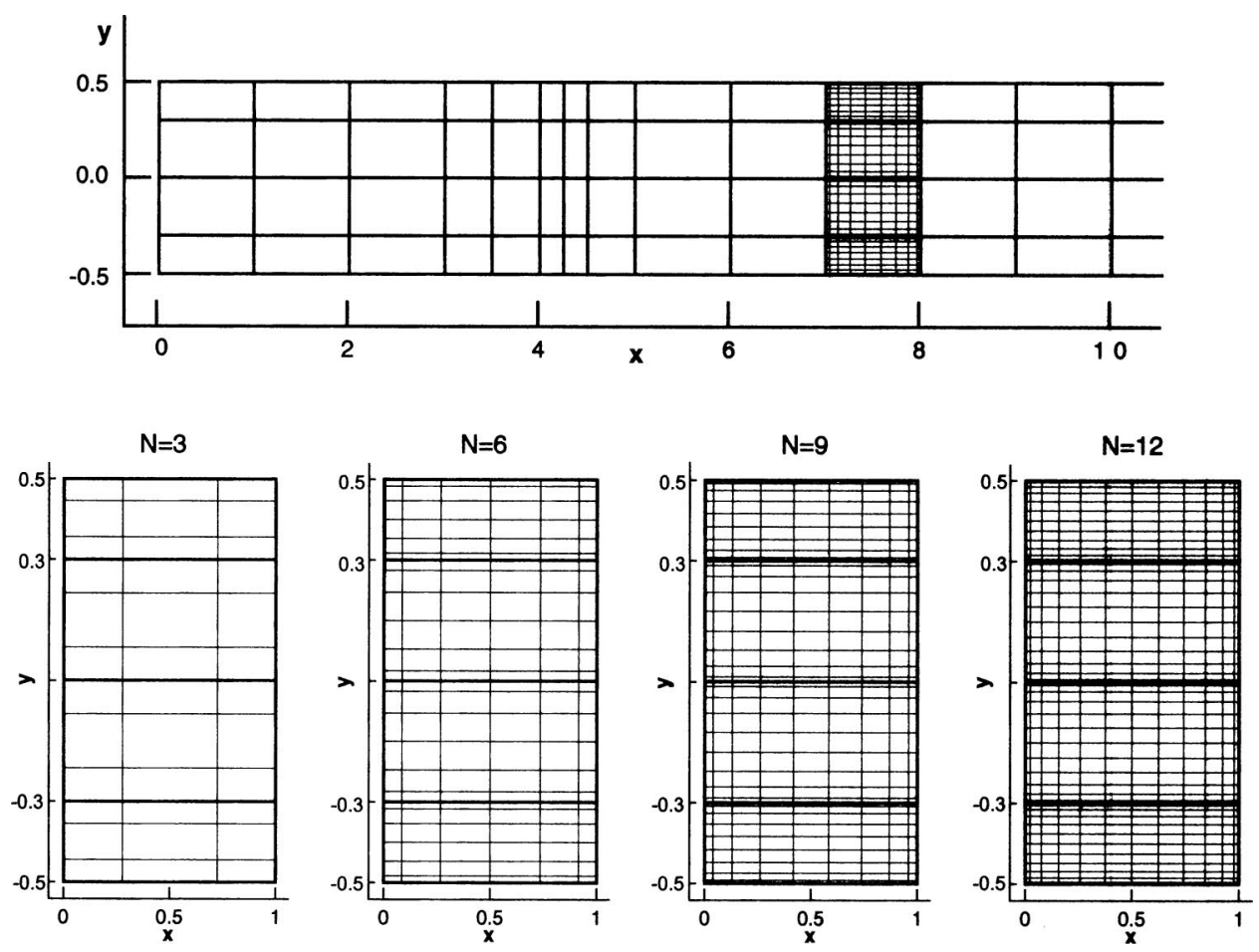

Fig. 4 Top: Half of the actual mesh used in the simulations. Quadrature points for a $9^{\text {th }}$-order expansion are also shown for selected elements. A finer mesh is used at the Neumann/Dirichlet boundary interface on the top wall $(4<x<4.5)$ to resolve large temperature variations. Bottom: A portion of the spectral element mesh showing only cross-channel discretization with different expansion orders $(\boldsymbol{N})$. Thick lines show the elements, while thin lines show the collocation points. Progressively increasing the element order $(N)$ by keeping the total number of elements fixed is known as $p$-type refinement.

method exhibits exponential reduction of discretization errors for sufficiently smooth $\left(C^{\infty}\right)$ problems upon $p$-type mesh refinement (Karniadakis and Sherwin [23]). The method also features small dispersion errors for long time integration of unsteady flow problems (Beskok and Warburton [24]).

We demonstrate the convergence characteristics of our method for $\alpha=10$ flow, which exhibits rather complex velocity profiles with large localized gradients. In Fig. 5(a), we show the variation of $L_{\infty}$ error norm (maximum error) as a function of the elemental expansion order $N$. The $L_{\infty}$ error is calculated using the numerical solution and the exact solution given in Eq. (9). The discretization error is evaluated by solving the unsteady problem with various spectral expansion orders, until a predetermined absolute time is reached. We utilized a very small time-step $\left(\Delta t=10^{-5}\right)$ in order to ensure that the leading order errors are due to spatial discreti-
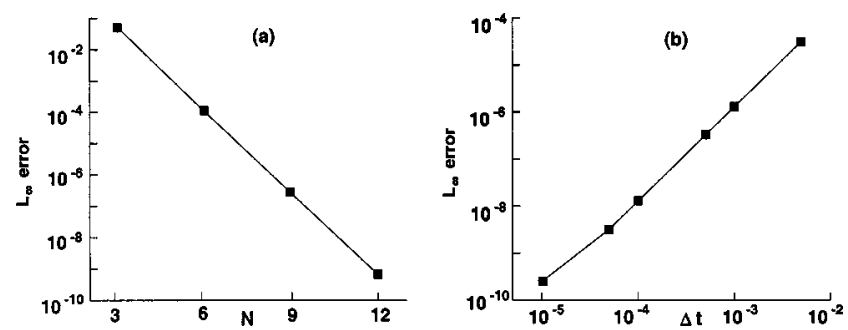

Fig. 5 Space and time accuracy for $\alpha=10$ flow. (a) Variation of $L_{\infty}$ error as a function of the expansion order $N$ (obtained using $\Delta t=10^{-5}$ ). Exponential decay of the discretization error indicates spectral convergence. (b) Variation of $L_{\infty}$ error as a function of the time step (obtained using $12^{\text {th }}$ order elements). Shows second-order time accuracy. zation. The results shown in Fig. 5(a) clearly indicate the spectral accuracy of the algorithm. Decrease of the discretization error by several orders-of-magnitude with $p$-type refinements is an important aspect of the spectral element method. For example in Fig. $5(a)$, the discretization errors of the $6^{\text {th }}$ and $12^{\text {th }}$ order expansions are about $10^{-4}$ and $10^{-9}$, respectively. This shows a reduction in the discretization error by five orders-of-magnitude, while the number of collocation points per direction is increased approximately by a factor of two (from 7 to 13 between the $6^{\text {th }}$ and $12^{\text {th }}$ order discretizations). Exponential decay of discretization errors by $p$-type refinements is a great advantage of spectral element methods over lower-order schemes, where grid independent solutions can be achieved more effectively.

Since we are solving a transient problem, time accuracy of the numerical algorithm also plays a significant role. Time accuracy is verified using controlled sets of simulations, in which, we employed $12^{\text {th }}$ order spectral elements to ensure that the leadingorder error in our solution is due to the time discretization. We integrated the transient problem with various time steps until a predetermined absolute time is reached. In Fig. 5(b), we show $L_{\infty}$ error variation as a function of the time step $\Delta t$. The slope of this $\log$-Log plot is 2, verifying that time accuracy of the algorithm is second-order. Since we do not have an analytical solution for the temperature field, we verified convergence of our numerical results by $p$-type mesh refinements, until grid independent solutions are obtained. The forthcoming results are obtained using polynomial orders of $7 \sim 9$ and $8 \sim 11$, for $\alpha=1$ and $\alpha=10$ cases, respectively.

\section{Results}

In this section we present detailed analyses of temperature field and heat transfer results for the cases presented in Table 1. We 


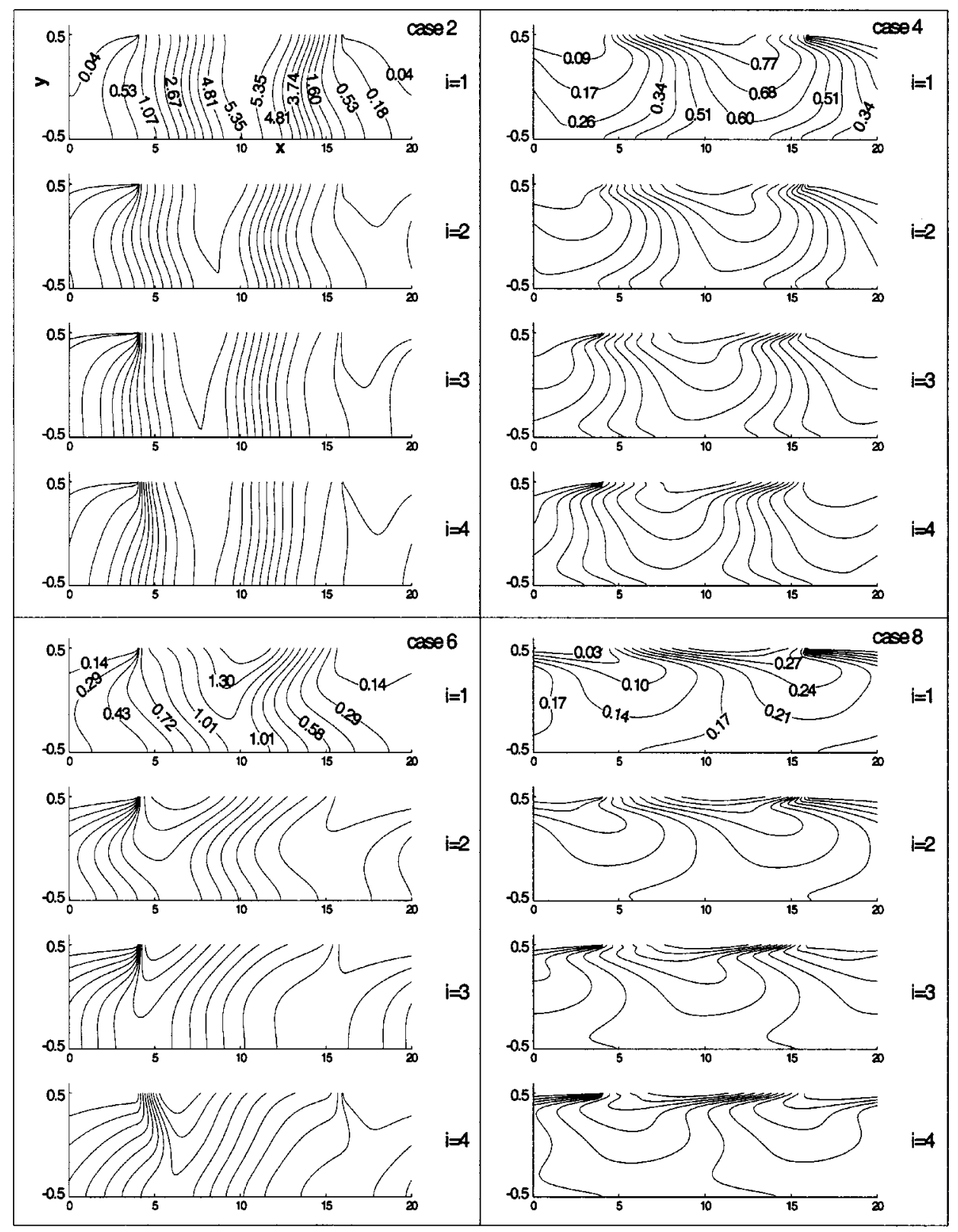

Fig. 6 Instantaneous temperature contours for cases 2, 4, 6, and 8 . Index $i$ represents time within half a period of the pressure pulse $(t=(i-1) \tau / 8)$. The flow and thermal conditions are presented in Table 1.

examine the effects of the Prandtl and the Womersley numbers as well as the tidal displacement on heat transfer. The results presented in the following sections are obtained after the simulations have reached their corresponding time-periodic states.

Temperature Contours. We present snapshots of temperature contours for cases 2, 4, 6, and $8(\mathrm{Pr}=10)$ in Fig. 6. These snapshots are synchronized with the pressure pulse, and they are obtained at $0 \tau, 1 / 8 \tau, 1 / 4 \tau$, and $3 / 8 \tau$. Comparisons of cases in the horizontal and vertical directions indicate the effects of $\alpha$ and $L_{p}$, respectively. The left column (cases 2 and 6) shows temperature contours oscillating back and forth with rather monotonic shapes. Especially for case 2, temperature contours across the channel are almost uniform at any time. Small $L_{p}$ and $\alpha$ values for case 2 result in the lowest axial velocities. Hence, conduction (in the axial direction) dominates over convection, as can be deduced from the temperature contours. For case 2, the hot fluid pocket under the heat source is not effectively convecting towards the cold walls. Case 6 has twice the tidal displacement of case 2, and it shows stronger cross-channel temperature variations. Values of the normalized temperature are significantly reduced from case 2 to case 6 , indicating increased convective cooling with increased tidal displacement $L_{p}$. Large cross-channel temperature variations are observed for cases 4 and 8 , which correspond to $\alpha=10$ flows. Case 8 has the highest $L_{p}$ and $\alpha$ values used in this work. Temperature contours for this case concentrate near the top surface, which indicates thin thermal boundary layers and enhanced forced convection. Temperature values are also significantly lower than the other cases. Our overall observation from Fig. 6 is that the normalized fluid temperature in the channels are decreased with increased tidal displacement $L_{p}$ and Womersley number $\alpha$, and the temperature distribution in the channel is highly affected by the velocity profile.

Temperature Profiles. Detailed descriptions of temperature profiles for reciprocating flows are not common in the literature. 


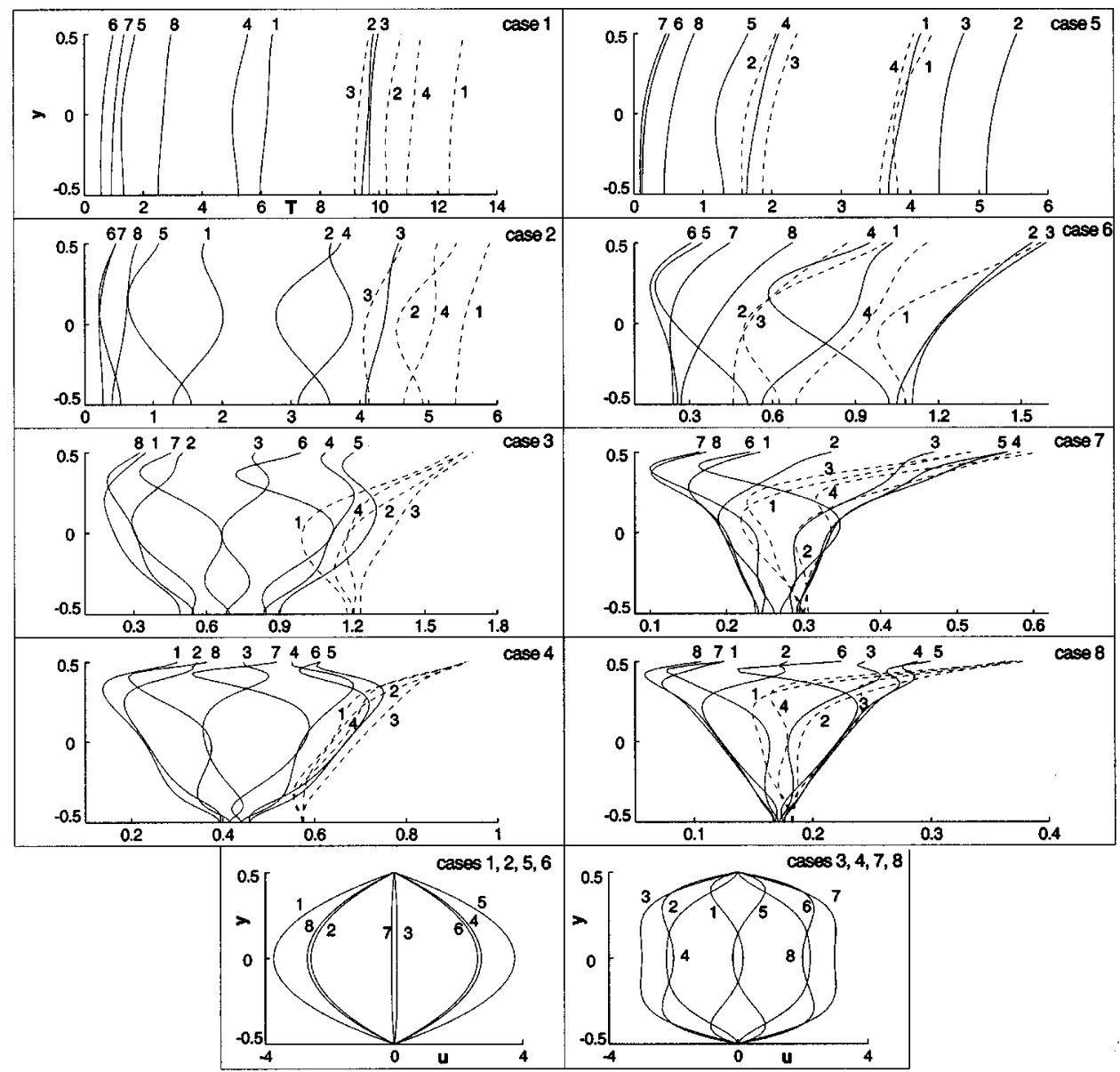

Fig. 7 Instantaneous temperature and velocity profiles at axial locations of $x=5$ (solid-lines) and $x=10$ (dashed-lines). Index $i$ represents time within a period of the pressure pulse $(t=(i-1) \tau / 8)$. Simulation parameters are presented in Table 1.

In Fig. 7, we present the temperature distribution at $x=5$ (solidlines) and $x=10$ (dashed-line) for all cases. Each figure shows the temperature profiles obtained at eight different instances. These snapshots are synchronized with the velocity profiles, which are shown at the bottom of the figure. The abscissa shows the temperature value, while the ordinate shows the cross-channel coordinate. The location $x=10$ corresponds to the geometric center of the channel, where non-dimensional heat flux of $q=1$ is imposed on the top wall. Due to the symmetry plane at $x=10$, temperature profiles at this location repeat twice cyclically from $i=1$ to 4 , while the temperature profiles at $x=5$ cycle from $i=1$ to 8 . In Fig. 7, the top four cases $(1,2,5$, and 6) are obtained under oscillatory parabolic velocity profiles $(\alpha=1)$, while the bottom four cases $(3,4,7$, and 8) are obtained for $\alpha=10$ flow. For $\alpha$ $=10$ cases, sharp velocity gradients near the walls result in enhanced oscillations in the temperature profiles. For example, the temperature profile in case 7 has as many as five inflection points at times $i=2,3,5,6$. It is clear from these results that the Richardson's annular effect, which exists in the velocity profiles at large frequencies, affects the temperature profiles. For case 1, temperature profiles are almost uniform at all times. An increase in the tidal displacement $L_{p}$ results in monotonic temperature variations, as shown in case 5 . In cases 1 and 2 , temperature values at $x=10$ are higher than the values at $x=5$, which is an indication of hot fluid being stuck under the heated region. The comparison of cases 6 and 7 in Fig. 7 shows that an increased Womersley number results in localized temperature gradients near the top wall with sudden temperature fluctuations. An increase in the Prandtl number creates sharper temperature variations in the cross-flow direction, as can be seen by a comparison of cases 7 and 8 . It is interesting to note that for a given axial location, the bottom wall temperature for cases 4,7 , and 8 remains almost constant throughout the cycle. All of these cases have a large $\alpha$ value, which corresponds to a large Re and enhanced convection. Heat supplied from the top plate rapidly convects along the channel and most of the thermal activity is occurring near the top wall. These cases result in bulk temperatures that are significantly lower than the surface temperatures, which results in high Nusselt numbers, as discussed in detail in the following sections.

Top-Wall Temperature Variations. In electronic cooling applications, exceeding a certain temperature may result in chip failure. Therefore, the maximum surface temperature is an important design parameter. In addition to the maximum surface temperature, the time of exposure to high temperatures also plays an important role. Figure 8 shows top wall temperatures at five different instances during half a cycle. Comparison of the left $\left(L_{p}=5\right)$ and right $\left(L_{p}=10\right)$ columns shows that the maximum top wall temperature for low penetration length simulations occurs in a narrowly bounded region near the channel center. However, for $L_{p}$ $=10$, the location of the maximum surface temperature is oscillating throughout the entire heated region. Comparisons of all eight cases show that the maximum surface temperature decreases with increased $L_{p}, \alpha$, and $\operatorname{Pr}$.

Bulk Temperature and the Nusselt Number. Bulk temperature is an important parameter, used in the calculation of the Nusselt number. Classical definition of the bulk temperature is 


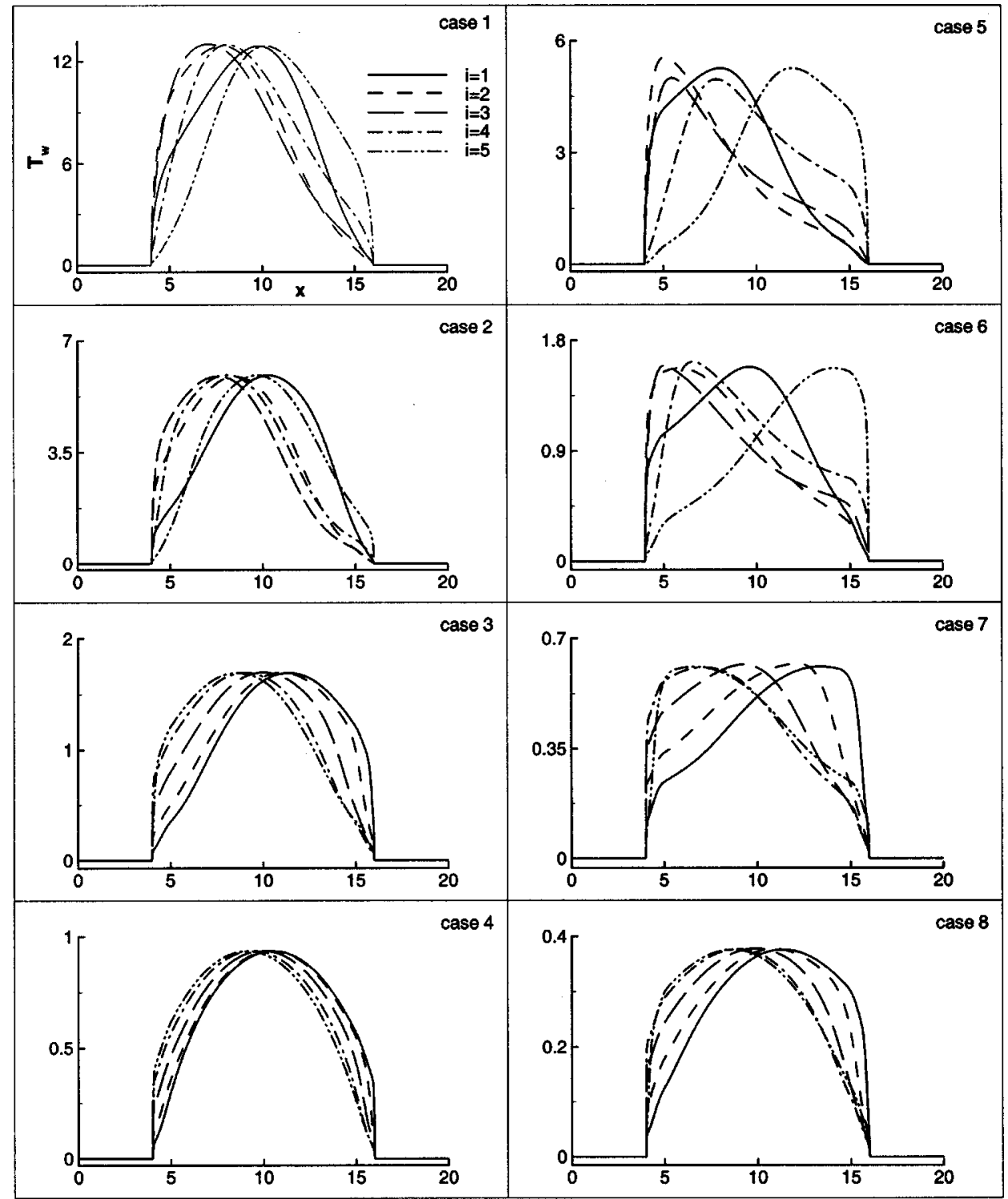

Fig. 8 Instantaneous top-wall temperatures. Index $i$ represents time within half a period of the pressure pulse $(t=(i-1) \tau / 8)$. Simulation parameters are presented in Table 1.

$$
T_{b}(x, t)=\frac{\frac{1}{H} \int_{-H / 2}^{H / 2} u(y, t) T(x, y, t) d y}{\frac{1}{H} \int_{-H / 2}^{H / 2} u(y, t) d y} .
$$

This definition is not preferred in a reciprocating flow, because the denominator becomes zero twice during a cycle. To overcome this difficulty, we defined a time-averaged bulk temperature in the following form,

$$
\bar{T}_{b}(x)=\frac{\frac{1}{\tau H} \int_{0}^{\tau} \int_{-H / 2}^{H / 2}|u(y, t)| T(x, y, t) d y d t}{\overline{\bar{u}}} .
$$

The absolute value in the numerator is used in order to avoid negative bulk temperatures during the flow reversal. An alternative definition can utilize time integration over a half cycle rather than a full cycle, which will yield the same result due to the half-period symmetry of the velocity and temperature fields.
Figure 9 shows axial variations of time-averaged top-wall (solid lines) and bulk (dashed lines) temperatures. The dashed-dotted and dashed-dotted-dotted lines in the figure correspond to unidirectional steady forced convection cases, which will be discussed in the next section. This figure shows that both the time-averaged wall temperature and bulk temperature decrease with increasing $L_{p}, \alpha$, and Pr. For $\alpha=1$ cases (top four plots), the bulk temperature values are close to the time-averaged wall temperature at 5 $<x<15$. This is especially noticeable for cases 1 and 2 . On the other hand, bulk temperatures for cases 7 and 8 are almost half of the time-averaged wall temperatures at $5<x<10$. Here we mention that cases 7 and 8 are the most effective in evenly spreading heat to the entire channel, as can be seen from their almost flat bulk temperature distributions. This also shows enhanced forced convection cooling for these cases.

In the simulations we specified a nondimensional constant heat flux value of unity in the region $5<x<15$. For $q=1$, the timeaveraged Nusselt number can be calculated as (Sert and Beskok [6]), 


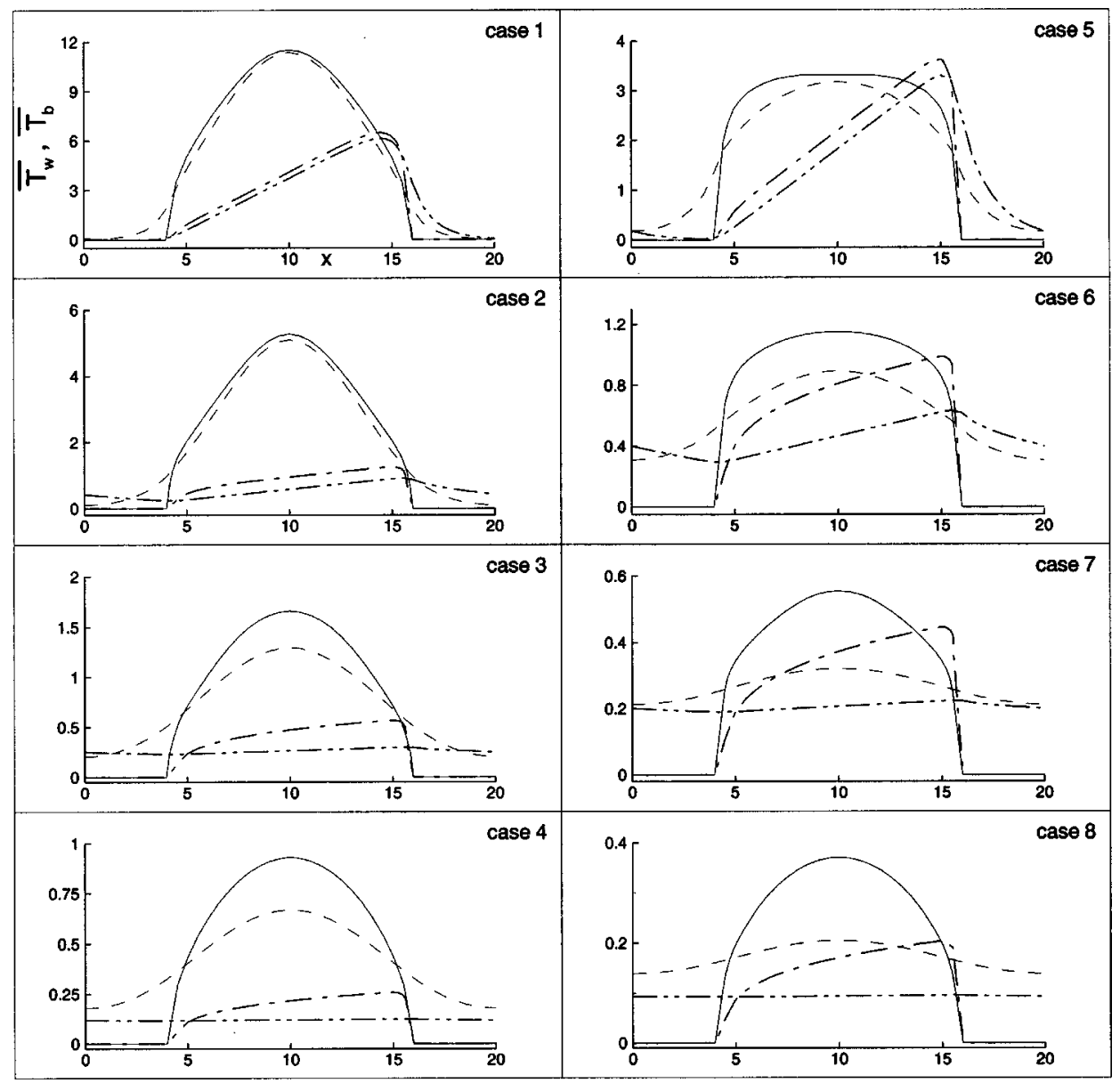

Fig. 9 Time-averaged wall-temperature (solid lines) and time-averaged bulk temperature (dashed-lines) variations for reciprocating flows. Wall temperature (dashed-dotted lines) and bulk temperature (dashed-dotted-dotted lines) variations for unidirectional steady flows are also shown. Simulation parameters are presented in Table 1.

$$
\overline{\mathrm{Nu}}(x)=\frac{2}{\bar{T}_{w}-\bar{T}_{b}} .
$$

Variations of time-averaged Nusselt number along the heated portion of the channel are shown in Fig. 10(a). We separated the low and high Womersley number cases. For $\alpha=1$ (cases 1, 2, 5, and 6 ), the maximum $\overline{\mathrm{Nu}}$ occurs at the middle of the channel ( $x$ $=10)$. For these cases, slight increases at $x=5$ and $x=15$ are due to the change in the boundary conditions (See Fig. 1). Specifically, the wall temperature suddenly drops to zero at $x=4$ and $x=16$, while the bulk temperature is decreasing gradually. These variations in the boundary conditions result in localized increases in the Nusselt number. It is interesting to notice that the tidal displacement and Prandtl number are both important here. Case 6 shows an almost uniform time averaged Nusselt number in the heated zone, which is an indication of effective heat transfer. The results for $\alpha=10$ are shown on the top left figure. For cases 3, 4, 7, and 8 , the maximum $\overline{\mathrm{Nu}}$ occurs at both ends of the heated region, whereas the minimum $\overline{\mathrm{N}} \mathrm{u}$ occurs at the channel center. Comparing case 3 with case 4 (or case 7 with case 8 ), we observe that the time averaged Nusselt number is increased by increasing the Prandtl number. Comparing case 3 with case 7 (or case 4 with case 8) shows that increasing the tidal displacement increases the Nusselt number. Finally, comparing cases 6 and 8 shows that $\overline{\mathrm{N} u}$ increases with the Womersley number.

Steady Unidirectional Forced Convection. In this section, previously obtained oscillatory flow temperature and Nusselt number results are compared against the steady, unidirectional forced convection. We match the oscillatory and unidirectional flows by matching the time-averaged flowrate. Reynolds number of the corresponding unidirectional flows is calculated by

$$
\operatorname{Re}^{\prime}=\frac{\overline{\bar{u}}^{*} H^{*}}{v^{*}}=\frac{\alpha^{2} L_{p}^{*}}{\pi H^{*}}
$$

which is presented in Table 1 . The velocity and temperature boundary conditions at the channel ends are periodic. Flow is from left to right, and it is maintained by a constant pressure gradient. The resultant velocity profiles are parabolic, typical of pressure driven laminar flows. Due to the periodic temperature boundary conditions, fluid leaving from the right boundary is entering from the left with a temperature equal to the exit temperature. Numerical results correspond to the steady state conditions.

Bulk and wall temperature variations along the channel are shown in Fig. 9 using dashed-dotted-dotted and dashed-dotted lines, respectively. For cases 1, 2, and 5, bulk and wall temperatures increase linearly with the same slope in most of the heated region, indicating thermally developed flow. We also observe that case 6 is almost thermally developed. Cases 1,2, 5, and 6 correspond to low Reynolds numbers $\left(\operatorname{Re}^{\prime}<4\right)$, resulting in relatively small Peclet numbers. Cases 3, 4, 7, and 8 correspond to Reynolds numbers that are two orders-of-magnitude higher than the corresponding low Reynolds number cases (See Table 1). This is due to the quadratic dependence of the Reynolds number on the Womer- 


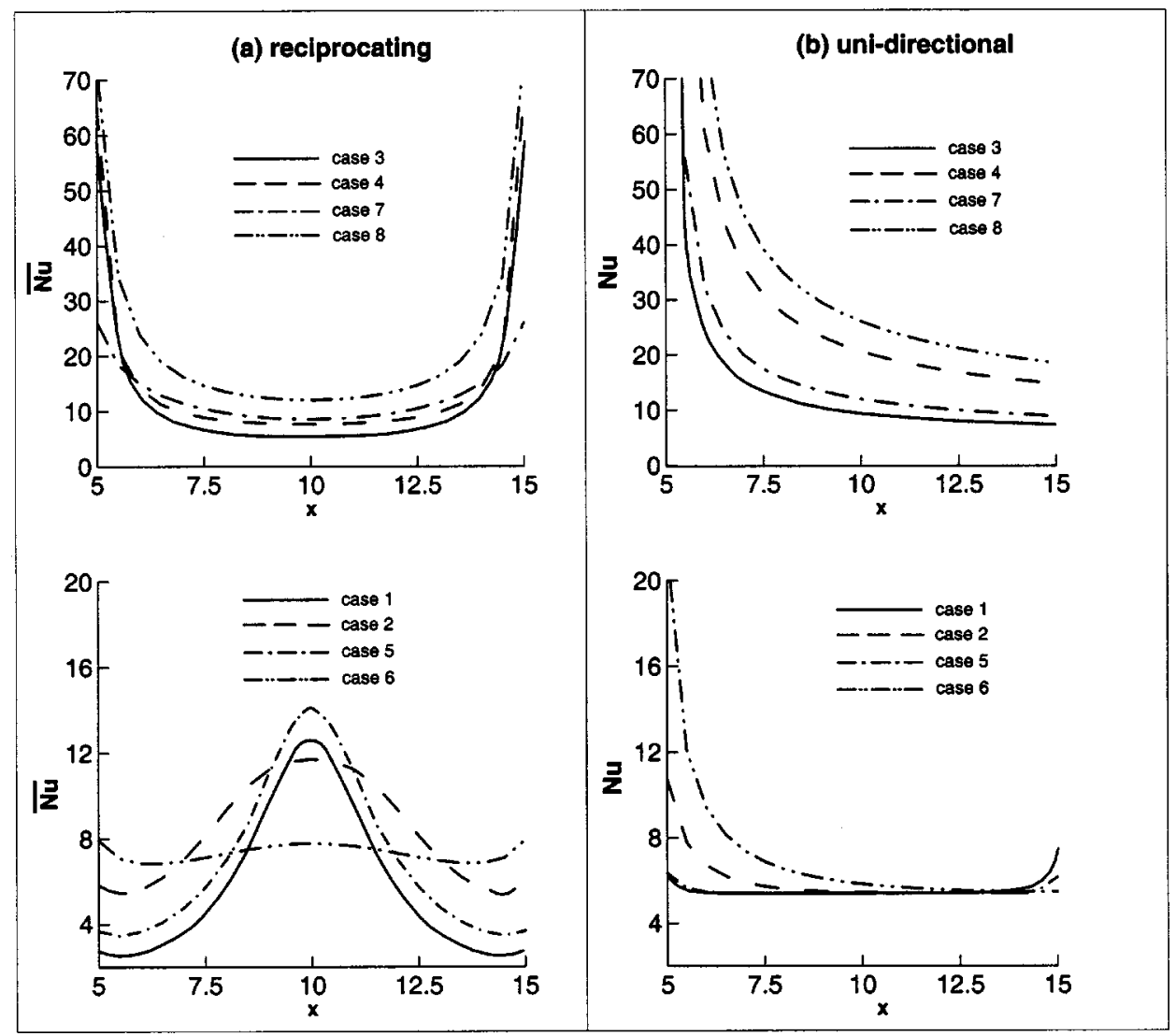

Fig. 10 Axial distribution of time-averaged Nusselt number for (a) reciprocating, (b) unidirectional steady flows. Simulation parameters are presented in Table 1.

sley number, given by Eq. (14). For these cases, thermally developed conditions are not observed. In all of the cases, unidirectional forced convection results in smaller bulk and surface temperatures than the reciprocating flow, with the exception of case 5 . In addition, we note that time-averaged top wall temperatures in Fig. 9 are less than the instantaneous maximum wall temperature shown in Fig. 8. Low $L_{p}$ cases (cases 1, 2, 3, and 4) give considerably higher maximum wall temperatures than their unidirectional flow counterparts. For cases 4 and 8, the bulk temperature of the unidirectional flows are almost uniform throughout the channel, and these cases correspond to the maximum Peclet numbers simulated in our work. Unidirectional flows result in increasing surface temperatures in the downstream direction, where oscillatory flows experience oscillating temperature maxima, as can be seen from Figs. 9 and 8, respectively. This basic difference may become important for various applications.

Nusselt number variations of unidirectional steady flows in the heated region are shown in Fig. 10(b). Maximum Nusselt number is observed at the entrance of the heated region and decreases continuously. Two exceptions are the slight increases seen at $x$ $=15$ for cases 1 and 5 . These cases have the lowest Pe, and local increase in the Nusselt number is due to noticeable heat conduction in the upstream direction. Cases 1,2, and 5 have reached thermally developed conditions with constant Nusselt number of 5.387, a value very close to 5.3846 given by Shah and London [1]. Cases 3, 4, 7, and 8 correspond to high Peclet numbers. For these cases, high Nusselt numbers are observed due to the thermally developing flow conditions.

\section{Discussion and Conclusions}

Motivated by its potential in electronic cooling and microfluidics applications, we simulated reciprocating flow forced convec- tion in two-dimensional channels, and compared our results with the corresponding unidirectional flows. We assumed a cyclically repeating flow section and imposed periodicity of velocity and temperature fields at the sides of the computational domain. To our knowledge, periodic thermal boundary conditions in reciprocating flows have not been investigated before. Simulation results indicate that the instantaneous and time-averaged surface temperatures, and the time-averaged bulk temperature are reduced by increasing the penetration length, Womersley and Prandtl numbers. Therefore, it is possible to determine a combination of $L_{p}$, $\alpha$, and Pr that will keep the maximum surface temperature below a desired value, which is important for cooling applications. Unlike unidirectional flows, reciprocating flows convect heat to both sides of the heated region. This results in oscillation of the maximum surface temperature along the heated region of the channel, which may be advantageous over unidirectional forced convection, where the maximum surface temperature occurs at the exit of the heated region.

Numerical simulations show an increase of the time-averaged Nusselt number with increasing $\alpha, \operatorname{Pr}$, and $L_{p}$. Variation of the time-averaged Nusselt number along the heated region of the channel shows different trends for $\alpha=1$ and $\alpha=10$ flows, indicating that velocity profiles have strong influences on the heat transfer characteristics. Richardson's annular effect, observed in the velocity profiles for high frequency oscillatory flows, affects the temperature profiles and heat transfer characteristics, as shown by the instantaneous temperature contours and profiles. Unidirectional flows corresponding to $\alpha=1$ cases show thermally fully developed conditions towards the downstream portion of the channel. For these cases, the reciprocating flow Nusselt numbers is comparable to or higher than the corresponding unidirectional flow cases. Overall unidirectional flows resulted in smaller surface 
temperatures than the reciprocating flows. However, we believe that with proper combinations of the parameters, heat transfer rates higher than the corresponding unidirectional flows can be achieved. In future studies, a larger parameter space will be explored by varying the heated length $\left(L_{h}\right)$ and the total length $(L)$ of the channel.

Finally, temperature dependence of fluid properties, viscous heating and three-dimensionality effects are not considered in the current study. Temperature dependence of fluid properties is significant for large temperature variations. This may be checked by calculating the maximum temperature difference $\Delta T^{*}$ obtained for a desired heat flux value. Viscous heating may become important for high Re flows. Three-dimensionality effects are important for turbulent channel flows; but all cases studied here, including the steady unidirectional flows, fall into the laminar flow regime.

\section{Acknowledgments}

This work was funded by Texas Higher Education Council, Advanced Research Program, Grant Number: 000512-0418-1999. The computational facilities provided by Dell STAR Grant are appreciated.

\section{Nomenclature}

$A=$ pressure gradient amplitude

$C_{p}=$ specific heat at constant pressure

$H=$ channel height

$k=$ heat conduction coefficient

$L=$ total channel length

$L_{p}=$ penetration length

$L_{h}=$ heated channel length

$\mathrm{Nu}=$ Nusselt number

$\mathrm{Pe}=$ Peclet number $(\operatorname{Re} \times \operatorname{Pr})$

$\operatorname{Pr}=$ Prandtl number $\left(\rho^{*} v^{*} C_{p}^{*} / k^{*}\right)$

$q=$ heat flux

$\operatorname{Re}=$ Reynolds number for oscillatory flows $\left(\omega^{*} H^{* 2} / v^{*}\right.$ $\left.\equiv \alpha^{2}\right)$

$\operatorname{Re}^{\prime}=$ Reynolds number based on volumetric flowrate per channel width $\left(\overline{\bar{u}}^{*} H^{*} / v^{*}\right)$

$t=$ time

$u=$ axial velocity

$x=$ streamwise direction

$y=$ cross-flow direction

Greek

$$
\begin{aligned}
\alpha & =\text { Womersley number }\left(\sqrt{\omega^{*} H^{* 2} / v^{*}}\right) \\
\nu & =\text { kinematic viscosity } \\
\tau & =\text { period } \\
\omega & =\text { frequency }
\end{aligned}
$$

\section{Subscript and Superscript}

$$
\begin{aligned}
b= & \text { bulk quantity } \\
o= & \text { reference value, side-wall value } \\
-= & \text { time-averaged quantity, used for top wall and bulk tem- } \\
& \text { peratures and Nu }
\end{aligned}
$$

$$
\begin{aligned}
& ==\text { time and space averaged quantity } \\
& *=\text { dimensional quantity }
\end{aligned}
$$

\section{References}

[1] Shah, R. K., and London, A. L., 1978, "Laminar Flow Forced Convection in Ducts," Advances in Heat Transfer, Irvine, T. F., Jr., and Hartnett J. P., eds. Academic Press, New York, NY.

[2] Zamir, M., 2000, The Physics of Pulsatile Flow, Springer-Verlag, New York, NY.

[3] Beskok, A., and Warburton, T. C., 2001, "Arbitrary Lagrangian Eulerian Analysis of a Bidirectional Micro-Pump Using Spectral Elements," Int. J. Comput. Eng. Sci. 2(1), pp. 43-57.

[4] Yi, M., Bau, H. H., and Hu, H., 2000, "A Peristaltic Meso-Scale Mixer," in Proceedings of ASME IMECE Meeting, MEMS, 2, pp. 367-374.

[5] Liao, Q. D., Yang, K. T., and Nee, V. W., 1995, "Microprocessor Chip Cooling by Channeled Zero-Mean Oscillatory Air Flow," Advances in Electronics Packaging, EEP-Vol. 10-2, pp. 789-794.

[6] Sert, C., and Beskok, A., 2002, "Oscillatory Flow Forced Convection in Micro Heat Spreaders," Numer. Heat Transfer, Part A. 42(7), pp. 685-705.

[7] Oddy, M. H., Santiago, J. G., and Mikkelsen, J. C., 2001, "Electrokinetic Instability Micromixing," Anal. Chem., 73-24, pp. 5822-5832.

[8] Dutta, P., and Beskok, A., 2001, "Time Periodic Electroosmotic Flows: Analogies to Stokes' Second Problem," Anal. Chem., 73(21), pp. 5097-5102.

[9] Siegel, R., and Perlmutter, M., 1962, "Heat Transfer for Pulsating Laminar Duct Flow," ASME J. Heat Transfer, 84, pp. 111-123.

[10] Siegel, R., 1987, "Influence of Oscillation-Induced Diffusion on Heat Transfer in a Uniformly Heated Channel," ASME J. Heat Transfer, 109, pp. 244-247.

[11] Kim, S. Y., Kang, B. H., and Hyun, J. M., 1993, "Heat Transfer in the Thermally Developing Region of a Pulsating Channel Flow," Int. J. Heat Mass Transf., 36, pp. 4257-4266.

[12] Moschandreou, T., and Zamir, M., 1997, "Heat Transfer in a Tube with Pulsating Flow and Constant Heat Flux," Int. J. Heat Mass Transf., 40, pp. 24612466.

[13] Zhao, T., and Cheng, P., 1995, "A Numerical Solution of Laminar Forced Convection in a Pipe Subjected to a Reciprocating Flow," Int. J. Heat Mass Transf., 38, pp. 3011-3022.

[14] Greiner, M., Fischer, P. F., and Tufo, H., 2001 "Numerical Simulations of Resonant Heat Transfer Augmentation at Low Reynolds Numbers," in Proceedings of ASME International Mechanical Engineering Congress and Exposition, IMECE2001/HTD-24100, November 11-16, 2001, New York, NY.

[15] Chatwin, P. C., 1975, "The Longitudinal Dispersion of Passive Contaminant in Oscillating Flow in Tubes," J. Fluid Mech., 71, pp. 513-527.

[16] Kurzweg, U. H., and Zhao, L., 1984, "Heat Transfer by High-Frequency Oscillations: A New Hydrodynamic Technique For Achieving Large Effective Thermal Conductivities," Phys. Fluids, 27, pp. 2624-2627.

[17] Kurzweg, U. H., 1985, "Enhanced Heat Conduction in Oscillating Viscou Flows Within Parallel-Plate Channels," J. Fluid Mech., 156, pp. 291-300.

[18] Li, P., and Yang, K. T., 2000, "Mechanisms for the Heat Transfer Enhancement in Zero-Mean Oscillatory Flows in Short Channels," Int. J. Heat Mass Transf., 43, pp. 3551-3566.

[19] Cooper, W. L., Nee, V. W., and Yang, K. T., 1994, “An Experimental Investigation of Convective Heat Transfer From the Heated Floor of a Rectangular Duct to a Low Frequency Large Tidal Displacement Oscillatory Flow,” Int. J. Heat Mass Transf., 37, pp. 581-592.

[20] Chou, F-C., Weng, J-G., and Tien, C-L., 1998, "Cooling of Micro Hot Spots by Oscillatory Flow," Proceedings of the $11^{\text {th }}$ International Symposium on Transport Phenomena, Hsinchu, Taiwan, pp. 324-329.

[21] Richardson, E. G., and Tyler, E., 1929, "The Transverse Velocity Gradient Near The Mouths of Pipes in Which An Alternating or Continuous Flow of Air is Established," Proc. Phys. Soc. London, 42, pp. 1-15.

[22] Landau, L. D., and Lifshitz, E. M., 1987, Course of Theoretical Physics Volume 6-Fluid Mechanics, $2^{\text {nd }}$ ed., Pergamon Press.

[23] Karniadakis, G. M., and Sherwin, S. J., 1999, Spectral/hp Element Methods for $C F D$, Oxford University Press, New York.

[24] Beskok, A., and Warburton, T. C., 2001, "An Unstructured H/P Finite Element Scheme for Fluid Flow and Heat Transport in Moving Domains," J. Comput. Phys., 174, pp. 492-509. 